Esta revista forma parte del acervo de la Biblioteca Jurídica Virtual del Instituto de Investigaciones Jurídicas de la UNAM

\title{
BRIAN BIX*
}

1. ¿Qué es la filosofía del derecho? ¿Cuál es su metodología adecuada?

"Filosofía del derecho" es el nombre que se da al amplio estudio filosófico de temas relacionados con el derecho. Su núcleo, creo, sigue siendo las investigaciones acerca de la naturaleza del derecho. No obstante, la "filosofía del derecho" es mucho más que eso: abarca desde las amplias y abstractas preguntas sobre la naturaleza o esencia del derecho, hasta las más detalladas discusiones sobre la naturaleza e interacción de varios conceptos jurídicos (por jemplo, la obligación jurídica, el deber jurídico, la causalidad sine qua non), incluyendo el análisis de los fundamentos filosóficos de diferentes áreas del derecho (por ejemplo, el derecho contractual o la responsabilidad extracontractual), y las investigaciones morales y políticas sobre los límites apropiados del derecho en general o del derecho criminal en particular.

En cuanto al propósito, las preguntas principales de la filosofía del derecho, las investigaciones sobre la naturaleza del derecho y la adecuada comprensión de los conceptos jurídicos centrales, no tienen propósito alguno más allá del saber en sí mismo. Aunque muchos teóricos jurídicos (angloparlantes) adoptan esta posición, es muy resistida por académicos de otros campos de estudio, en especial, me parece, por los académicos estadounidenses. En su opinión, casi todas las teorías tienen implicaciones prácticas, y si no las tienen, no merecen nuestra atención. En ocasiones, esto hace difícil presentar artículos sobre filosofía del derecho a las audiencias estadounidenses.

(Debo añadir aquí que hay un prominente teórico del derecho angloparlante que reivindica una estrecha relación entre la teoría jurídica propia y las consecuencias jurídicas del día a día; ese es Ronald Dworkin. Él cree que la resolución de cada disputa jurídica específica implica o expresa una opinión sobre temas generales de la jurisprudencia, y que cada posición sobre temas generales de la jurisprudencia tendrá como consecuencia diferentes resultados en muchas discusiones jurídicas particulares).

* University of Minnessota Law School.bix@umn.edu 
Esta revista forma parte del acervo de la Biblioteca Jurídica Virtual del Instituto de Investigaciones Jurídicas de la UNAM

En cuanto a la metodología, no hay razones para asumir que hay una sola metodología correcta para todos los diferentes temas e investigaciones que caben dentro de la filosofía del derecho. Por ejemplo, el análisis de la naturaleza del derecho o de la naturaleza de los derechos jurídicos podría fácilmente requerir un diferente conjunto de herramientas metodológicas que las necesarias para determinar los fundamentos filosóficos de la responsabilidad extracontractual.

Por otra parte, aun en lo relacionado con la naturaleza del derecho, uno podría aproximarse al derecho (como muchos teóricos del derecho natural lo hacen) como un aspecto de la razón práctica, o uno podría aproximarse (como muchos positivistas lo hacen) como un tipo de práctica social. El enfoque teorético con el que uno se aproximaría a un aspecto del razonamiento moral se diferencia muy probablemente del enfoque con el que uno se aproxima a una práctica social.

2. ¿Cuál es su experiencia personal? ¿Cómo comenzó en la filosofía del derecho? ¿Qué personas le influenciaron sustancialmente en su trabajo?

Durante mi primer año como estudiante de pregrado en la Washington University, tuve la buena fortuna, primero, de ser parte de un pequeño grupo cuyos cursos se centraron en el tema "derecho y sociedad" - el cual incluía la teoría jurídica, la historia del derecho y la antropología jurídica- y, segundo, de tener como uno de mis profesores en ese grupo a Stanley Paulson, el gran experto en Kelsen.

Después de graduarme en la Washington University (con una licenciatura en filosofía y ciencia política, y un título adicional en biología), obtuve mi título de abogado en la Harvard University. Mientras estaba allí, tuve la oportunidad de hablar y aprender de algunos de los más importantes teóricos del movimiento de los estudios críticos del derecho (CLS), entre los cuales estaban Duncan Kennedy, Morton Horwitz, Roberto Unger, Gerald Frug y David Kennedy.

(Nunca he sido un teórico crítico del derecho; sin embargo, he tratado de aprender del movimiento de los CLS. Esa es mi actitud hacia muchas escuelas de pensamiento jurídico. Puede que no esté de acuerdo con ellas, pero sus opiniones contienen verdades importan- 
Esta revista forma parte del acervo de la Biblioteca Jurídica Virtual del Instituto de Investigaciones Jurídicas de la UNAM

tes de las cuales puedo aprender. Creo que eso es cierto, no sólo para los CLS, sino también para la teoría del derecho natural, la teoría crítica raciales, el movimiento de derecho y economía, el realismo estadounidense, el realismo escandinavo, la jurisprudencia histórica, y muchos otros enfoques).

Luego, en el transcurso de mi trabajo doctoral en Oxford University, tuve la oportunidad de tener mi tesis dirigida por Joseph Raz, y de tomar cursos con Ronald Dworkin, John Finnis y J. W. Harris. También, mientras estudiaba en Oxford, tuve la ocasión de atender conferencias y clases de figuras importantes en el estudio de Wittgenstein, la filosofía del lenguaje y la filosofía moral; por ejemplo, Michael Dummett, Simon Blackburn, Crispin Wright, Bernard Williams, John McDowell, David Pears, G. P. Baker y Peter Hacker.

Junto con los anteriores teóricos, tuve la fuerte influencia de $\mathrm{H}$. L. A. Hart (en especial, el enfoque del sentido común inglés, y la claridad en el lenguaje y en los argumentos de su obra), Ludwig Wittgenstein (especialmente su esfuerzo por disolver los problemas de la filosofía en general y aquellos del significado en particular), y Robert Gordon (el académico de los estudios críticos del derecho y de la historia jurídica).

3. ¿Cuáles son las áreas y temas en los que ha trabajado en filosofía jurídica? ¿Cuáles han sido sus mayores influencias?

Muchos de mis escritos en jurisprudencia encajan en una de estas tres categorías: 1) la relación entre la filosofía del lenguaje y la filosofía del derecho; 2) temas metodológicos relativos a teorías acerca de la naturaleza del derecho, y 3) la naturaleza de la verdad en el derecho.

Yo no puedo reivindicar ninguna escuela de pensamiento particular o ideas grandes y novedosas. Mi trabajo, principalmente, ha sido contribuir al trabajo colectivo de muchos; por ejemplo, al entendimiento y defensa de las teorías sobre la naturaleza del derecho como teorías conceptuales, a aclarar el (limitado) rol que las teorías de la filosofía del lenguaje pueden jugar en temas como el razonamiento jurídico, la interpretación jurídica y la determinación jurídica, y a investigar la naturaleza de la verdad en el derecho. En 
Esta revista forma parte del acervo de la Biblioteca Jurídica Virtual del Instituto de Investigaciones Jurídicas de la UNAM

relación con la filosofía del lenguaje y la filosofía del derecho, creo que es importante no caer en la tentación de subestimar el rol que la política (entendida como elección colectiva, aunque arbitraria) juega y debería jugar en el derecho. En las discusiones sobre la naturaleza de la verdad de las proposiciones jurídicas, me he centrado en dos temas: a) contrastar e intersecar los roles de la "voluntad" y la "razón" al justificar la verdad de las proposiciones jurídicas, y b) las dificultades conceptuales de afirmar un error global acerca de una cuestión jurídica.

4. ¿Cuál es el futuro de la filosofía del derecho? ¿Qué problemas piensa que deberían recibir mayor atención en los próximos años? ¿Cuáles cree usted que son las formas más útiles de aproximarse a estos problemas?

Preveo que el futuro de la filosofía del derecho no será muy diferente del presente: una lucha teórica para obtener una más clara visión general de la naturaleza del derecho, de la naturaleza de conceptos jurídicos como derecho, deber, causalidad y dominio, además de los fundamentos filosóficos de la propiedad, el derecho contractual, la responsabilidad extracontractual, entre otros temas.

Hay una continua controversia sobre si hay (alguna vez) progreso en la filosofía, o si sólo nos movemos de una teoría de moda a la siguiente, de un conjunto de términos oscuros y mitificantes a otro. Creo que ha habido progreso de alguna manera en la teoría jurídica en las décadas recientes - en parte, porque creo que el punto de referencia ha sido muy bajo-. Hay personajes destacados en las primeras décadas — quienes todavía merecen ser leídos-, pero quienes, para nosotros, parecen terriblemente desprovistos de sofisticación y de herramientas teoréticas. Por ejemplo, con John Austin (el primer positivista jurídico moderno y quizá el primer filósofo del derecho analítico), uno no puede distinguir en el texto si las afirmaciones que hace son descriptivas, conceptuales o algo más. Los prominentes realistas estadounidenses Karl Llewellyn y Jerome Frank se esfuerzan al máximo para hacer afirmaciones sobre la naturaleza del lenguaje y las reglas, y sobre los efectos económicos de las reglas jurídicas, pero ellos no tenían las herramientas para tra- 
Esta revista forma parte del acervo de la Biblioteca Jurídica Virtual del Instituto de Investigaciones Jurídicas de la UNAM

bajar sobre sus ideas con cuidado. Además, las tesis de Lon Fuller acerca de la justicia procesal, el estado de derecho y la competencia institucional tienen que ser extraídas de un análisis que con demasiada frecuencia es confuso y desafortunado.

Hay un impresionante grupo de teóricos jurídicos trabajando que incorporan sofisticadas herramientas teóricas de la filosofía del lenguaje, el razonamiento práctico, la sociología, la economía, los estudios culturales, la teoría literaria y muchas otras disciplinas. Creo que hay muchos motivos para pensar que habrá una constante corriente de trabajos excelentes que incorporarán diversos métodos interdisciplinarios y ofrecerán nuevas ideas y perspectivas.

Aunque dentro de la teoría jurídica, cada generación plantea diferentes grupos de preguntas según las preocupaciones de sus sociedades y su época, creo que ciertos problemas básicos todavía siguen sin ser contestados y merecerán una mayor atención; por ejemplo, el fundamento de la verdad de las proposiciones jurídicas (por ejemplo, qué hace que sean falsas o verdaderas afirmaciones como "A y B tienen un contrato válido" $\mathrm{y}$ "X tiene un derecho a que $Y$ le page $\$ 400 ”$ ), la adecuada comprensión de la lógica deóntica, en sí misma y aplicada a las normas jurídicas, el mejor entendimiento de los diferentes vínculos entre el derecho y la moral, y si podemos hablar con sentido de "la naturaleza del derecho".

Lo que quisiera enfatizar es la complejidad de la investigación: por ejemplo, uno no debería centrarse en el aspecto del razonamiento práctico del derecho mientras se ignora el aspecto de las prácticas sociales. Además, uno no debería centrarse en el aspecto de la filosofía del lenguaje aplicada al razonamiento jurídico mientras se olvida el rol que cumplen tanto la lógica deóntica como la elección política. 